\title{
Exenatide Response in an Adolescent's Morbid Obesity
}

\author{
Ricardo Soriano Sánchez, Alfonso López Ruiz*, M. Ángeles Núñez Sánchez, \\ Noelia Victoria García-Talavera Espín, Amparo Meoro Avilés, \\ María Teresa Gallego García, Cristina del Peso Gilsanz, José Soriano Palao, \\ Collaborative Groups of the Diabetes and Obesity Unit of the Hospital Reina Sofía \\ Unidad de Diabetes y Obesidad del Área VII Murcia Este, Hospital General Universitario Reina Sofía de Murcia, \\ Murcia, España \\ Email: ${ }^{\text {alfonsolopezruizmail@gmail.com }}$
}

Received 11 February 2014; revised 23 March 2014; accepted 10 April 2014

Copyright (C) 2014 by authors and Scientific Research Publishing Inc.

This work is licensed under the Creative Commons Attribution International License (CC BY).

http://creativecommons.org/licenses/by/4.0/

(c) (i) Open Access

\begin{abstract}
Adolescent obesity is a health problem worldwide. Drug therapy for obesity in children is only acceptable if done within the framework of a comprehensive weight loss, prevention of comorbidities, including health education, changes in lifestyles, diet and exercise. These pharmacological treatments indicate that mainly young patients did not respond to standard measures and developing complications. The role of bariatric surgery, with or without the use of drugs is subject to further research to elucidate its role among the various treatment options for these patients. Currently being studied a wide range of drugs to those included gastrointestinal hormones. Scientific information regarding the use of exenatide in obese patients without diabetes is still limited. The use of exenatide in adolescents with morbid obesity may represent a therapeutic complement invaluable, effective and free of complications, and prevents future bariatric surgery.
\end{abstract}

\section{Keywords}

Type 2 Diabetes Mellitus; Exenatide; Adolescent; Obesity; Rational Use of Drugs; Pharmaceutical Care

\section{Introduction}

Worldwide, more than 42 million children under five years are overweight, which has become one of the largest public health problems of this century. Overweight children are likely to become obese adults and more likely to

"Corresponding author.

How to cite this paper: Sánchez, R.S., Ruiz, A.L., Sánchez, M.Á.N., Espín, N.V.G.-T., Avilés, A.M., García, M.T.G., del Peso Gilsanz, C., Palao, J.S. and Collaborative Groups of the Diabetes and Obesity Unit of the Hospital Reina Sofía (2014) Exenatide Response in an Adolescent's Morbid Obesity. Pharmacology \& Pharmacy, 5, 419-424.

http://dx.doi.org/10.4236/pp.2014.54051 
develop diabetes as well as cardiovascular disease at younger ages, which are associated with an increase in premature death and disability.

Obesity is one of the most important preventable causes of death. The factors that modulate the morbidity and mortality associated with obesity are age of onset, duration, severity, comorbidities, sex, race, and respiratory functional capacity.

Obesity in children and adolescent has reached epidemic proportions in Spain. It is important of the investment in research on interventions for primary prevention and treatment of obesity and the development of well-designed studies on a large scale in this area to identify effective treatments and risk groups.

It is known that central fat distribution (visceral) shows a genetic predisposition, with associated environmental and hormonal factors and the adult weight gain occurs with greater accumulation of fat in this location, which is associated with intolerance to carbohydrates or type 2 diabetes mellitus (T2DM), hypertriglyceridemia and hypertension. In obese children and adolescents, fat deposition occurs mainly in the central region as in the adult. In infants and very young children, obesity associated with these complications is rare, however, in adolescence mainly due to increased growth and others hormones, it has the appearance of a physiological insulin resistance and we do observe these disorders. Currently, obesity is defined as the most important risk factor for developing type 2 diabetes mellitus classic. $80 \%$ of these diabetics are significantly overweight, insulin resistance syndrome and compensatory insulin response, and favoring the links in the beginning of this type of diabetes is triggered by obesity. This condition is sometimes seen in obese children, so the diagnosis should be considered before a patient with diabetes associated with obesity [1]. In the other hand, type 2 diabetes mellitus in children and adolescents (in $90 \%$ of cases are associated with obesity) includes many abnormalities including insulin resistance, increased hepatic glucose production, abnormal production of hormones, including insulin, glucagon, and incretins.

Incretin hormones are peptides derived from the gut, functions in glucose regulation. Incretin dysfunction can be treated with agonists of glucagon-like peptide-1 (exenatide). GLP-1 is a glucose-dependent insulinotropic peptide produced by the $\mathrm{L}$ and $\mathrm{K}$ endocrine cells of the intestine after ingestion of food. Exenatide is a synthetic version of exendin-4 that has some homology with the amino acid pattern of GLP-1, but has a half life in vivo over prolonged by what has been considered effective in the diabetic state. It also has an appetite suppressant effect.

Exenatide $\left(\right.$ Byetta $\left.^{\circledR}\right)$ is a medication approved in 2005 for the treatment of type 2 diabetes mellitus. It has been used as an adjunctive therapy to improve glycemic control in patients with T2DM taking met form alone or in combination with sulfonylurea, which have not achieved their glycemic control. Exenatide helps control glucose in several ways:

1) Increase the response pancreatic (insulin release) in response to food intake.

2) Suppress glucagon release in response to meals, avoiding sugar the liver to overproduce when not needed, which prevents hyperglycemia.

3) Slow gastric emptying, reducing the amount of glucose from food that appears in blood.

4) Reduce appetite and promote satiety through hypothalamic receptors. Most patients using exenatide lost weight slowly and generally greater weight loss is achieved in those with greater overshoot at the start of treatment. It has been shown that the weight reducing effect continues for more than two years of continuous use.

5) Reduce the fat content of the liver, especially in patients with DM2.

But there is not (so much) experience in non-diabetic adolescent morbid obese patients treated with exenatide in order to lose weight.

\section{Case Presentation}

A 17-year-old male caucasian patient. Consultation on type III obesity. Weight: 142.5 kg, Size: 171.5 m, BMI: $48.2 \mathrm{~kg} / \mathrm{m}^{2}$, Tanner V, $20 \mathrm{ml}$ testicular volume, initial waist/hip 120/115. After despistation on secondary causes of obesity with normal levels of tirotrophyn hormone $(1.59 \mathrm{mcU} / \mathrm{ml})$, free tiroxine $(1.34 \mathrm{mg} / \mathrm{dl})$ and 24 hours urinary free cortisol $(51 \mathrm{mcg})$ treatment with Exenatide 5 micrograms/bid and 10 micrograms/step-by-dose + Orlistat $120 \mathrm{mg}$ in lunch and dinner $+1200 \mathrm{kcal}$ equilibrated (15\% protein, 30\% fat, 55\% carbohidrates) diet + $60 \mathrm{~min} /$ day bid aerobic exercise.

The patient history of surgery was apendicectomy and amigdalectomy. He was studying informatica. School achievement.

Newborn weight: $2750 \mathrm{~kg}$. Progressive increase in weight from 6 years ago related to inactivity and adaptive problems and social relationship due to obesity. 
Family history: obesity in the father (weight $85 \mathrm{~kg}$, size $175 \mathrm{~cm}, \mathrm{BMI} 27.76 \mathrm{~kg} / \mathrm{m}^{2}$ ), mother (weight $65 \mathrm{~kg}$, size $159 \mathrm{~cm}$, BMI $25.71 \mathrm{~kg} / \mathrm{m}^{2}$ ) and brother (weight $65 \mathrm{~kg}$, size $159 \mathrm{~cm}$, BMI $25.71 \mathrm{~kg} / \mathrm{m}^{2}$ ). Good family support.

Combination therapy with exenatide and orlistat achieved a $57.5 \mathrm{~kg}$ loss without adverse effects, except bloating, in an adolescent with morbid obesity (Table 1).

\section{Discussion}

The treatment of obesity is normally based on changes in patients' lifestyle: proper nutrition and exercise. It is known that weight loss and physical activity, lead to an improvement in lipids levels, insulin-resistance and blood pressure. But in young children, weight loss should not be aimed because it can affect growth. They must "stall weight", so, as it grows, it will go back to a normal weight.

When it comes to teenagers, low-calorie diets to reduce weight in fat should be individualized according to patient's eating patterns, level of motivation and family support. The diet should be balanced to provide all necessary nutrients (55\% complex carbohydrates, $30 \%$ fat and $15 \%$ proteins). For the reduction of caloric intake is taken into account the patient's weight, growth rate, degree of adiposity, desired weight and daily physical activity. Very low calories diets are not recommended in children. Our patient failed to lose weight by this conventional treatment until the age of $17^{\text {th }}$.

In general, any treatment will have disappointing results, and $80 \%$ of obese adolescents will be obese adults, hence the importance of prevention using more aggressive treatments.

In secondary prevention programs, treatment outcomes are better when obese children are treated by the obese parent, intervention strategies at home, by promoting changes in lifestyle of the family, produced encouraging results and should be considered in any program if you want to achieve success in this aspect of primary health care will play an important role. Our patient had significant family support, as parents also followed the

Table 1. Response to exenatide plus orlistat in an adolescent with morbid obesity.

\begin{tabular}{|c|c|c|c|c|}
\hline & Initial & 3 Months & 6 Months & 12 Months \\
\hline Weight (kg) (Figure 1) & 142.5 & 131 & 103.5 & 85 \\
\hline$\Delta$ Weight $(\mathbf{k g})$ & 0 & -11.5 & -39 & -57.5 \\
\hline BMI $\left(\mathrm{kg} / \mathrm{m}^{2}\right)$ & 48.2 & 44.3 & 34.8 & 31.8 \\
\hline Waist Perimeter (cm) & 120 & 114 & 107 & 98 \\
\hline Glucose (mg/dl) (Figure 2) & 83 & 84 & 86 & 78 \\
\hline Creatinine (mg/dl) & 0.79 & 0.79 & 0.78 & 0.83 \\
\hline GOT/GPT/GGT (U/l) & 29/39//39 & $38 / 53 / 27$ & $35 / 41 / 115$ & $26 / 22 / 14$ \\
\hline Tryglicerides (mg/dl) & 126 & 118 & 87 & 64 \\
\hline HDL-Cholesterol (md/dl) & 45 & 41 & 57 & 59 \\
\hline LDL-Cholesterol (mg/dl) & 90 & 92 & 98 & 82 \\
\hline Total Cholesterol (mg/dl) & 160 & 157 & 172 & 154 \\
\hline HbA1c (\%) (Figure 3) & 4.5 & 4.5 & 4.5 & 4.8 \\
\hline Albumin (g/dl) & 4.7 & 4.6 & 5 & 5 \\
\hline Ketonuria & 5 & Negative & Negative & Negative \\
\hline Blood Pressure (mmHg) & $141 / 83$ & $139 / 80$ & $138 / 80$ & $129 / 80$ \\
\hline Ferritin (ng/ml) & 90 & 92 & 93 & 96 \\
\hline Vitamin B12 (pg/ml) & 340 & 346 & 343 & 345 \\
\hline Folate (ng/ml) & 10.23 & 10.12 & 10.67 & 10.46 \\
\hline
\end{tabular}




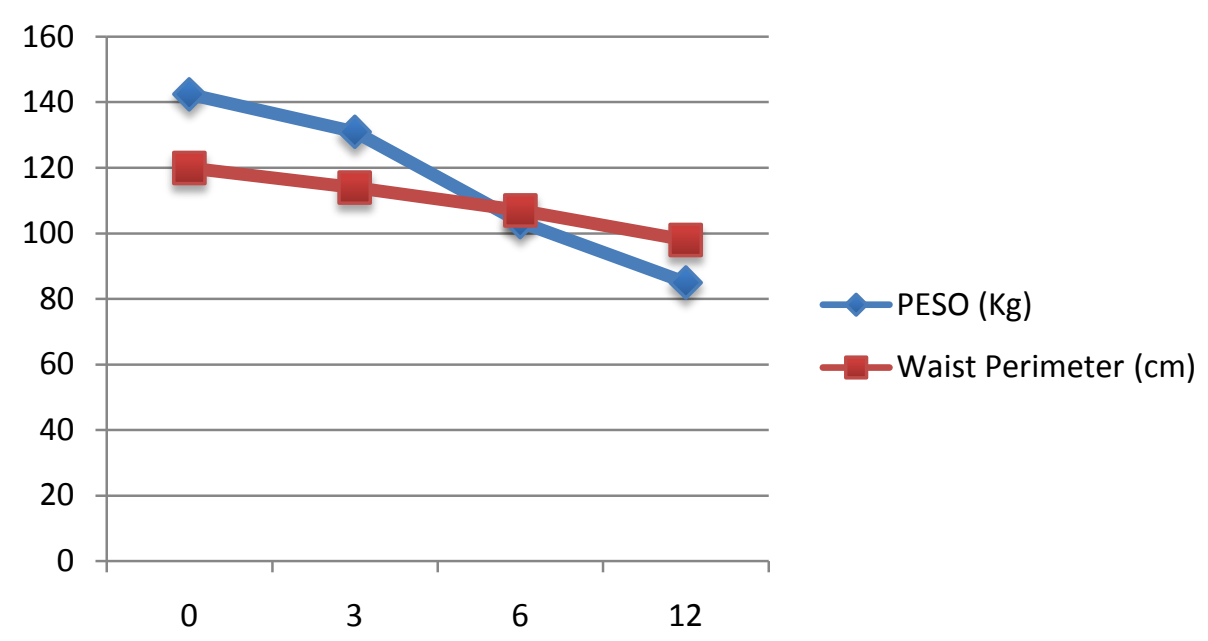

Figure 1. Weight vs time (month).

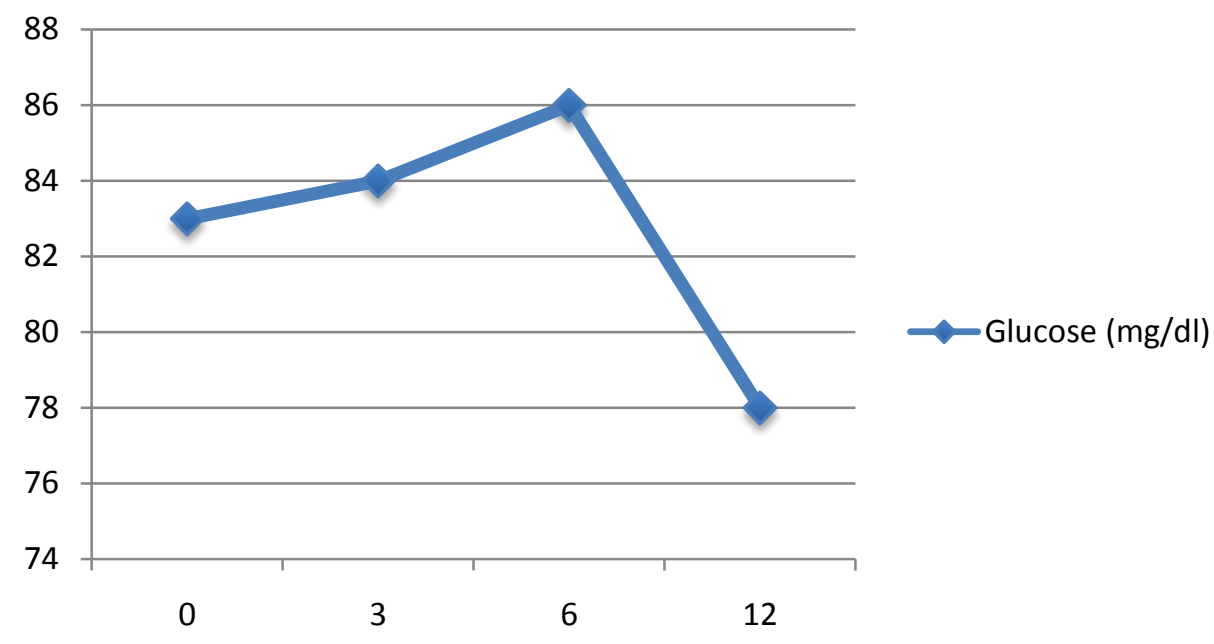

Figure 2. Glucose vs time (month).

HbA1c vs time (month)

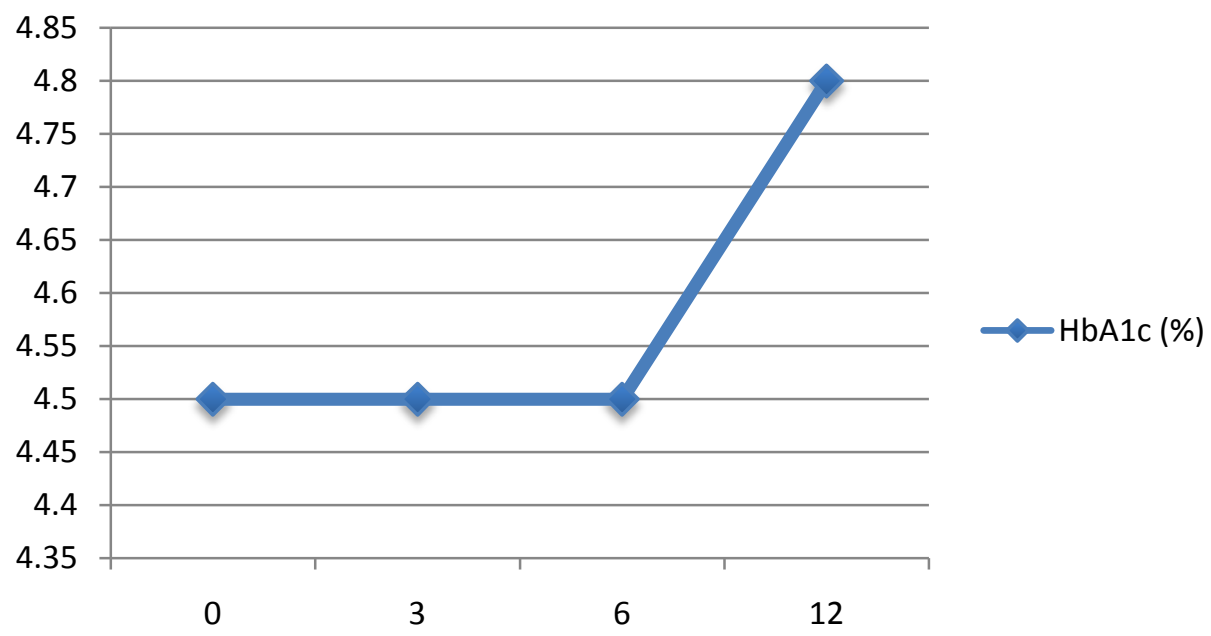

Figure 3. HbA1c vs time. 
diet and even lost weight. Strategies for preventing obesity should have the collaboration of multiple sectors of the community who are sensitized to the problem and primary health care [2].

There are many opportunities to conduct research to identify the best option for the treatment of obesity in children and adolescents [3]. The causes of the obesity epidemic are complex. Pharmacologic therapies for the treatment of obesity are an important area of scientific interest. Although in adults there are several categories of approved drugs, the studies are limited in children and adolescents especially in terms of safety and efficacy. A recent meta-analysis conducted by the Endocrine Society found that there are 17 trials of pharmacological agents for the treatment of pediatric obesity [4]. These include orlistat and sibutramine, among others. Each of these drugs has a specific role in populations of obese adolescents in addition to changes in lifestyle. Some Korean researchers have advocated the safety and short-term effectiveness of a Korean traditional formulation of herbal based on Taeumjowi-tang for children an average of 11 years. This formula is a popular herbal inducing weight loss in children, adolescents and adults. It consists of a mixture of roots, berries, nuts and seeds. One component is the Ephedra sinica contains alkaloids ephedrine and pseudoephedrine.

Bariatric surgery improves obesity and its comorbidities in adults with morbid obesity complicated but the data in pediatrics are limited. There are rates of up to $10 \%$ of reoperations, so the safety of this type of surgery should be considered. Recommendation is considered premature for this type of treatment in the adolescent population, suggesting that they should only be considered after surgical treatment failure of a multidisciplinary management, appropriate weight control at least six months. Another aspect to consider is the high cost of this alternative health treatment. The role of bariatric surgery in this group of patients, with or without the help of certain drugs is yet to be elucidated.

Treatment options for obesity, insulin resistance, metabolic syndrome and type 2 diabetes in young people includes fully changes in lifestyle and careful consideration of medical or surgical treatment. However, the evidence is still limited. Of the other diabetes drugs, only metformin is associated with weight loss. The obesity and associated metabolic disorders treatment in children remains an area of scientific interest, ripe for future research. The safety, effectiveness and long-term consequences of these drugs in young obese are unclear. The use of these drugs should be part of a comprehensive, complete, comprehensive emphasizes proper diet, exercise and changes behavior.

There are new potential pharmacological approaches to treat obesity in young people. There is a large and growing array of research for the management of obesity.

The incretin-like, in particular exenatide, are analogues of GLP-1 that modulate the effects of prandial glucagon, limiting hyperglycemia and stimulating insulin secretion decreased gastric emptying and also cause satiety, suppressing food intake, improving glycemic control and weight loss in patients with type 2 diabetes, a loss that can be breathtaking and maintained. There were no reported serious adverse events related to treatment. Those not in favor are based on the high cost of medication and gastrointestinal side effects.

In a randomized study of 551 diabetic patients treated with exenatide was associated with greater weight loss, but these had more gastrointestinal symptoms such as nausea, vomiting and diarrhea [5]. What is sometimes interpreted as nausea is a feeling of fullness. Most patients have less need to eat and until fit to eat smaller portions, the result is the feeling of satiety. If this therapy proves effective in achieving sustained weight loss is yet to be demonstrated. Nausea is an early and rate-limiting effect in a large group of patients with diabetes, but symptoms disappear over time in some patients.

Reduce the fat content of the liver, especially in patients with DM2. It has been used successfully exenatide in Prader Willi Syndrome, a genetic disorder that presents with severe obesity, because it produces satiety and no side effects were reported.

Our patient showed an excellent response to treatment with exenatide and orlistatin terms of weight loss and lack of side effects (hypoglycemia, biochemical, developmental and maturational statural ponder...), similar to the weight loss after bariatric surgery in adolescents and without the risks that it entails.

The use of exenatide in obese patients without diabetes remains a subject for study and debate. The use of exenatide in adolescents with morbid obesity may represent a therapeutic complement invaluable.

\section{Conclusion}

Adolescent obesity is a health problem worldwide. Drug therapy for obesity in children is only acceptable if done within the framework of a comprehensive weight loss, prevention of comorbidities, including health edu- 
cation, changes in lifestyles, diet and exercise. These pharmacological treatments indicate that mainly young patients did not respond to standard measures and developing complications. The role of bariatric surgery, with or without the use of drugs is subject to further research to elucidate its role among the various treatment options for these patients. Currently being studied a wide range of drugs to those included gastrointestinal hormones. Scientific information regarding the use of exenatide in obese patients without diabetes is still limited. The use of exenatide in adolescents with morbid obesity may represent a therapeutic complement invaluable, effective and free of complications, and prevents future bariatric surgery.

\section{References}

[1] Lamas Piñeyro, R. (2006) Obesity in Children and Adolescents Topics in Pediatrics. Valdés, M.S. and Vasallo, G.A., Eds. Medical Sciences, Havana.

[2] US Food and Drug Administration (2008) CDER Drug and Biologic Approvals for Calendar Year 2005.

[3] Buncke, M.C., Diamant, M., Corner, A., Eliasson, B., Malloy, J.L., Shaginian, R.M., et al. (2009) One-Year Treatment with Exenatide Beta-Cell Function Improves, Compare with Insulin Glargine, Metformin in Type 2 Diabetic-Treat Patients: A Randomized, Controlled Trial. Diabetes Care, 32, 762-768. http://dx.doi.org/10.2337/dc08-1797

[4] Tushuizen, M.E., Buncke, C.M., Pouwels, P.J., van Waesberghe, J.H., Diamant, M. and Heine, R.J. (2006) Incretinmimetics as a Novel Option for Hepatic Steatosis Therapeutic. Liver International, 26, 1015-1017. http://dx.doi.org/10.1111/j.1478-3231.2006.01315.x

[5] Heine, R.J., Van Gaal, L.F., Johns, D., Mihm, M.J., Wideload, M.H., Brodows, R.G., et al. (2005) Exenatide versus Insulin Glargine in Suboptimally Controlled Type with Patients 2 Diabetes: A Randomized Trial. Annals of Internal Medicine, 143, 559-569. http://dx.doi.org/10.7326/0003-4819-143-8-200510180-00006 